\title{
Size Complexity of Volume Meshes vs. Surface Meshes
}

\author{
Benoît Hudson Gary L. Miller Todd Phillips \\ Computer Science Department \\ Carnegie Mellon University \\ \{bhudson, glmiller, tp517\}@cs.cmu.edu
}

Oct 26, 2007. Fall Workshop on Computational Geometry.

\begin{abstract}
Typical volume meshes in three dimensions are designed to conform to an underlying two-dimensional surface mesh, with volume mesh element size growing larger away from the surface. The surface mesh may be uniformly spaced or highly graded, and may have fine resolution due to extrinsic mesh size concerns. When we desire that such a mesh have good aspect ratio, we require that some space-filling scaffold vertices be inserted off the surface. We analyze the number of scaffold vertices in a setting that encompasses many existing volume meshing algorithms. We show that for surfaces of bounded variation, the number of scaffold vertices will be linear in the number of surface vertices.
\end{abstract}

\section{Introduction}

The mesh refinement problem is to take an input geometry in $\mathbb{R}^{d}$, and produce a partition into output cells that in some sense respects the input. Inputs are often point clouds, usually representing samples of an implicit surface to reconstruct. Inputs may also be given as piecewise linear complexes (PLCs), assemblages of segments and polygons. The output is a mesh, where the points appear as mesh vertices, and the segments and polygons appear as the union of mesh segments and mesh facets.

The meshing problem also requires a constraint on the quality of the output elements. We use the constraint that the output vertices must be well-spaced [8]; all the Voronoi cells in the Voronoi diagram of the output vertices must have aspect ratio bounded by some constant. Additionally, the elements must be small enough to capture the geometry of the surface, or perhaps even smaller to capture some extrinsic properties. Algorithmically, we then wish to produce a mesh with the fewest elements subject to these constraints. This general setting encompasses the output of most volume or surface meshing algorithms having any provable guarantees, including (at least) $[7,5,4,3,1]$.

A natural question when analyzing the output of such algorithms is to consider the number of vertices placed on

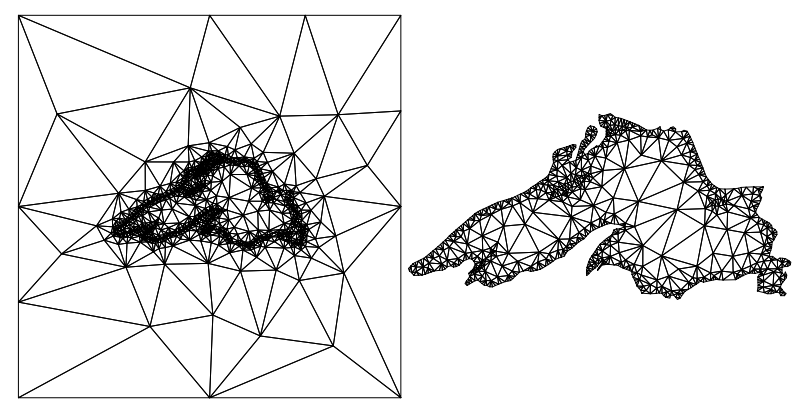

Figure 1: Often, mesh refinement algorithms first generate a mesh over a bounding box (left), then remove the scaffold vertices and elements. (right). We analyze the cost of removing this scaffolding for the first time.

the input surface compared with the number of vertices in space. Often the surface vertices are the main source of interest, and the extraneous scaffold vertices merely support this structure by filling space.

Additionally, in incremental algorithms, usually a reasonably-sized bounding box is used, with the mesh being generated within this box. In this case, some portion of the scaffold vertices may be wholly outside the domain of interest (see Figure 2). We would like the number of these scaffold vertices to be as small as possible.

It is intuitive that the scaffolding should be small, since the complexity of the mesh is generated at the surface. We conjecture that the total number of scaffold vertices (interior and exterior to the domain) is linear in the number of surface vertices.

\section{Definitions}

We present a generic, algorithm-independent setting for analyzing volume meshes of a surface. The goal of this setting is to abstractly analyze several modern volume meshing algorithms at once. Consider a connected Piecewise Smooth Complex [1] $\mathcal{S}$ in some simply connected volume $\Omega \subset \mathbb{R}^{3}$. We require that $\Omega$ not be too large (or we could not control the volume mesh size). The easiest assumption is that the diameter of $\Omega$ is bounded within a constant of the diameter of $\mathcal{S}$. 
We define a sizing function $f$ on $\mathcal{S}$ as any function having the following three properties:

(1) $f$ is 1-Lipschitz:

$$
\forall x, y \in \mathcal{S}, f(x) \leq f(y)+|x-y|_{\mathbb{R}^{3}}
$$

(2) $f$ is bounded from below:

$$
\exists f_{0}, \forall x \in \mathcal{S}, f(x)>f_{0}>0
$$

(3) $f$ "captures" $\mathcal{S}$. Defining:

$$
I(x):=\left\{y \in \mathcal{S}|| x-\left.y\right|_{\mathbb{R}^{3}} \leq f(x)\right\}
$$

Then there must exists some constant $K$ for all $x$ that bounds $|I(x)| / f^{2}(x)$, and $I(x)$ must be a topological 2-ball.

We then define an extension $f_{e}$ on $\Omega$ as the maximum 1-Lipschitz function on $\Omega$ that agrees with $f$ on $\mathcal{S}$. For some algorithms, the function $f$ may be given explicitly. Examples of such an $f$ in meshing include varying definitions of local feature size. Epsilon sampling algorithms may have an $f$ that represents some diminished feature size. Adaptive remeshing algorithms used in simulation may have an $f$ that is reduced according to a simulation error estimate.

If $M$ is a point set, we say that $M$ is $f$-sized if it is wellspaced, and if the nearest neighbor of any $m \in M$ satisfies $|N N(m)-m|_{\mathbb{R}^{3}} \in \theta\left(f_{e}(m)\right)$.

\section{Proof Sketch}

For an $f$-sized pointset $M$, we have:

$$
|M| \in \theta\left(\int_{\Omega} \frac{1}{f_{e}^{3}}\right)
$$

If $N \subset M$ are the vertices whose Voronoi cells intersect $\mathcal{S}$, then we have a similar measure:

$$
|N| \in \theta\left(\int_{\mathcal{S}} \frac{1}{f^{2}}\right)
$$

These measures are generic and well-established in several settings $[6,5,8]$, where they have previously been used to argue the size-optimality of meshing algorithms, although we are considering a more generic non-optimal case (governed by a more arbitrary $f$ ).

We conjecture that in general $|M| \in O(|N|)$. We argue this by comparing the two integrals in question. The general case is still elusive, but for the special case of a convex $\mathcal{S}$, we can show that:

$$
\int_{\Omega} \frac{1}{f_{e}^{3}} \in O\left(\int_{\mathcal{S}} \frac{1}{f^{2}}\right)
$$

which completes the argument.

\section{Notes}

Our proof technique is probably applicable to $\mathcal{S}$ and $\Omega$ in any dimensions, with constants that are necessarily exponential in dimension. Possiblities include meshing a curve in $\mathbb{R}^{3}$ or perhaps a surface in space time.

The "capturing" condition on $f$ is rather crude. It is necessary for establishing Equation 2 and is coupled to whatever smoothness assumptions are taken on $\mathcal{S}$. This condition bears a strong relation to the traditional openball criterion in surface reconstruction [2]. For a more/less generic $\mathcal{S}$, this criterion could probably be refined. However, the main obstacle seems to be in proving Equation 3 for any reasonably general setting.

Because the constants involved in these equations come from packing arguments, they are generally unrealistic. Nonetheless, this result and conjecture are very interesting from a theoretical standpoint. Were this conjecture shown to be true, it would be an important philosophic point that volume mesh is asymptotically no more expensive than surface meshing. At the very least, it would confirm the intuition that external scaffolding is not too expensive, which has so often been implicitly assumed in prior work.

\section{References}

[1] S.-W. Cheng, T. K. Dey, and E. A. Ramos. Delaunay refinement for piecewise smooth complexes. In SODA '07: Proceedings of the eighteenth annual ACM-SIAM symposium on Discrete algorithms, pages 1096-1105, Philadelphia, PA, USA, 2007. Society for Industrial and Applied Mathematics.

[2] H. Edelsbrunner and N. R. Shah. Triangulating topological spaces. IJCGA, 7:365-378, 1997.

[3] B. Hudson, G. Miller, and T. Phillips. Sparse Voronoi Refinement. In Proceedings of the 15th International Meshing Roundtable, pages 339-356, Birmingham, Alabama, 2006. Long version available as Carnegie Mellon University Technical Report CMU-CS-06-132.

[4] G. L. Miller, S. E. Pav, and N. J. Walkington. Fully Incremental 3D Delaunay Refinement Mesh Generation. In Eleventh International Meshing Roundtable, pages 75-86, Ithaca, New York, Sept. 2002. Sandia National Laboratories.

[5] S. A. Mitchell and S. A. Vavasis. Quality mesh generation in higher dimensions. SIAM J. Comput., 29(4):1334-1370 (electronic), 2000.

[6] J. Ruppert. A Delaunay refinement algorithm for quality 2-dimensional mesh generation. J. Algorithms, 18(3):548585,1995 . Fourth Annual ACM-SIAM Symposium on Discrete Algorithms (SODA) (Austin, TX, 1993).

[7] J. R. Shewchuk. Tetrahedral Mesh Generation by Delaunay Refinement. In Proceedings of the Fourteenth Annual Symposium on Computational Geometry, pages 86-95, Minneapolis, Minnesota, June 1998. Association for Computing Machinery.

[8] D. Talmor. Well-Spaced Points for Numerical Methods. $\mathrm{PhD}$ thesis, Carnegie Mellon University, Pittsburgh, August 1997. CMU CS Tech Report CMU-CS-97-164. 